\title{
Dynamic Bayesian Belief Network for Modeling Student Knowledge Tracing
}

\author{
Habeebullah Saheb Shaik ${ }^{1}$, N. Sharada ${ }^{2}$ \\ ${ }^{1}$ M-Tech, Department of Computer Science and Systems Engineering, Andhra University, Visakhapatnam, Andhra Pradesh \\ ${ }^{2}$ Research Scholar, Department of Computer Science and Systems Engineering, Andhra University, Visakhapatnam, Andhra Pradesh
}

\begin{abstract}
E-learning offers an important feature to conventional learning by providing optimized learning. For this, accurate representation of student's current skills and adaptation to newly acquired skills are essential. In this, we show that the development of skills in children for those who are having difficulties in learning arithmetic skills is possible through graphical models by evaluation of data gathered from different children by conducting tests. The model consists of Dynamic Bayesian Network which incorporates domain knowledge and identifies the difficulties. The system posts appropriate tasks and exercise actions on the basis of estimated levels of gathered knowledge. The concepts that are believed to have been learned or not represent the evidence. Based on the evidence it is concluded that which concepts to be relearned and which not.
\end{abstract}

Keywords: E-learning, Dynamic Bayesian Network, domain knowledge, graphical models, Intelligent Tutoring System

\section{Introduction}

Education is a never ending process and has become one of the essential needs for every human being. Without proper education it is clear that the progress cannot be achieved. Information technology has become a major breakthrough in the field of education. IT has enabled a concept called elearning. The concept of e-learning has many advantages in learning and teaching process. This concept not only reduces the cost but also opens up opportunities to explore different models in different fields.

One special class of e-learning is Intelligent Tutoring System (ITS). By this we can say that computer has become student's personal teacher. It has the ability to model each student based on his competence and responses to questions to achieve improvement in learning process. The selection of probabilities is based on the student's knowledge. The key feature of these systems is the adaptation of learning content and the difficulty level to the individual student's knowledge. ITS are successfully employed in different fields of education particularly to overcome the learning disabilities.

Current tutoring systems use different approaches to assess and predict student's performance. Two of the most popular approaches for estimating student's knowledge are

- Performance factor analysis and

- Bayesian Knowledge Tracing.

The ITS simulates student-friendly teaching-learning environment to learn at our pace and interested specific domain knowledge. As ITS has become student's personal teacher, the computer teacher being inanimate has complete balance on one side can always be cheerful, instant negative emotions, while a student on the other hand has no need to hide ignorance and can communicate freely.

The Intelligent tutoring systems can adapt the content and manner of presentation according to different student abilities. In this sense, knowledge is the key to intelligent behavior and therefore ITS stores and maintains have the following types of knowledge:

- Specific domain knowledge that the system has to train the student.

- Teaching principles and the methods for applying these principles.

- Methods and techniques for modeling students during the process of learning.

Arithmetic skills are necessary in day-to-day life. Many children suffer from difficulties in learning mathematics. Developmental Dyscalculia (DD) is a specific learning disability. DD is a brain based disorder, although poor teaching and environmental deprivation might also be relevant. Computer based learning systems have the potential to offer an inexpensive extension to conventional education by providing a fear-free learning environment.

To provide effective teaching, adaptation to the student ${ }^{\text {es }}$ knowledge and capabilities is essential. This is particularly important for students suffering from learning disabilities. A variety of methods are currently employed to model student's knowledge and behavior. Calcularis [1] is a training program for children with Developmental Dyscalculia or difficulties in learning mathematics. It consists of multiple games in a hierarchical structure. The games are structured according to number ranges and can be divided into two areas. The first are focuses on number representation and number understanding in general. The second area focuses on addition and subtraction.

The components of an ITS that represents the students state of knowledge and skills is called the student model. The student model is a data structure and diagnosis is a process that manipulates the student model based on his responses during learning sessions. The student model as such represents a key component of an ITS. The design of this component is called the student modeling problem. Design and implementation of ITS systematically contributed to the

\section{Volume 4 Issue 11, November 2015}




\section{International Journal of Science and Research (IJSR) \\ ISSN (Online): 2319-7064}

Index Copernicus Value (2013): 6.14 | Impact Factor (2014): 5.611

development of methods and techniques of Artificial Intelligence (AI).

One of the techniques widely used in different areas of AI are Bayesian belief networks. Unlike traditional expert systems, where the main purpose is modeling the experts knowledge to simulate experts decision making and analyzing, the purpose of Bayesian network is modeling a particular problem domain by relating the causes and consequences of an action.

The domain modeling refers to collecting and determining all necessary values for Bayesian network initialization. Specially, it refers to modeling dependencies between variables. Dependencies are modeled using a network structure and set of conditional probabilities. Integration of student models with Bayesian network in the ITS is one way to facilitate student learning. This model allows making conclusions about the actual student knowledge. Also, it enables a computer tutor to guide the learning and teaching process towards the learning of only those concepts that the student has not already learnt.

\section{Methodology}

In our approach we enable non-empirical mathematical determination of conditional probabilities, while "a priori" probabilities are empirically determined based on the knowledge test results. The Bayesian network is a probabilistic model that displays dependencies between nodes. It is a Directed Acyclic Graph (DAG) in which nodes represent variables and edges represents their interdependencies. A node is a parent of a child if there is edge from the former to the latter. In Bayesian network nodes that have no parents are called roots.

After the structure of Bayesian network is defined, it is necessary to define the possible values of each node can take and the values of conditional probabilities of each possibility. For nodes without parents only, a-proirie probabilities have to be defined. The dimension of the conditional probability table is determined by parents of the node. The Bayesian network can be used for probabilistic inference on the probabilities of any node in the network if conditional probability tables are known. Based on the network the system can calculate the expectations of all unknown variables based on the known variables.

The mathematical knowledge of the student is modeled using dynamic Bayesian network. The network consisting of directed acyclic graph representing different mathematical skills and their mutual dependencies. Each skill has two states learnt state $\&$ unlearnt state. The probabilities for these states are inferred by posing tasks and evaluating user actions. After each solved task the system updates the posterior probabilities of the skills based on the current state three possible actions can be selected.[3]

- Going back to an easier skill,

- Going forward to more difficult skill or,

- Additional training of the current skill.
The decision is based on the upper and lower thresholds.

Two skills $\mathrm{S}_{\mathrm{A}} \& \mathrm{~S}_{\mathrm{B}}$ have a connection if mastering skill $\mathrm{S}_{\mathrm{A}}$ is a prerequisite for skill $\mathrm{S}_{\mathrm{B}}$. As the skills cannot be directly observed, the system infers them by posing tasks and evaluating user actions. For example, if a child can compute addition involving a ten crossing, we assume that the child also knows addition without ten crossing. The basic assumption is that to know skill $\mathrm{S}_{\mathrm{A}}$, the child needs to know all the precursor skills of $\mathrm{S}_{\mathrm{A}}$.

Since a directed acyclic graph represents a hierarchical arrangement, the goal is to calculate the posterior conditional probability distribution of each of the possible unobserved causes given the observed evidence i.e. $\mathrm{p}$ [cause | evidence]. The student model consists of a directed acyclic graph representing different mathematical skills and their relationships, the skills are connected based on the dependencies among them i.e. two abilities $\mathrm{A}$ and $\mathrm{B}$ have a connection, if having ability $\mathrm{A}$ is a precondition for having ability B. As the skills cannot be observed directly, the system infers them by posing specific tasks and evaluating user actions. Therefore, we assign all types of tasks and their outcome to different skills.

Computing $13+24=37$ (addition 2, 2) is considered [2] more difficult than calculating $5+2=7$ (addition 1,1 ) as the latter task involves smaller numbers. Furthermore, a task involving bridging to ten such as $38+6=44$ (addition 2, 1 with bridging to ten) is rated more complex than a task without any crossing. Finally, modeling the task $13+2=15$ (addition 2,1) is easier than calculating the task mentally (addition 2, 1).

All probabilities are initiated to 0.5 as the system doesn $i t$ know anything about the knowledge of the child. The probabilities are updated after each trial. The probability of a skill can be influenced in different ways. On one hand, it changes if the child solves a task that is associated with this skill. On the other hand, solving a task that is associated with a precursor or a successor skill influences the probability.

The basic assumption of the model is that in order to pass the current skill, all the precursor skills need to be mastered. The decision is based on an upper and lower border for the probability of the current skill. If the probability is larger than the upper border a more difficult skill is selected. If it is smaller a precursor skill is selected. In order to pass a skill about 8 tasks out of 10 has to be solved correctly otherwise it leads to failing the skill. 


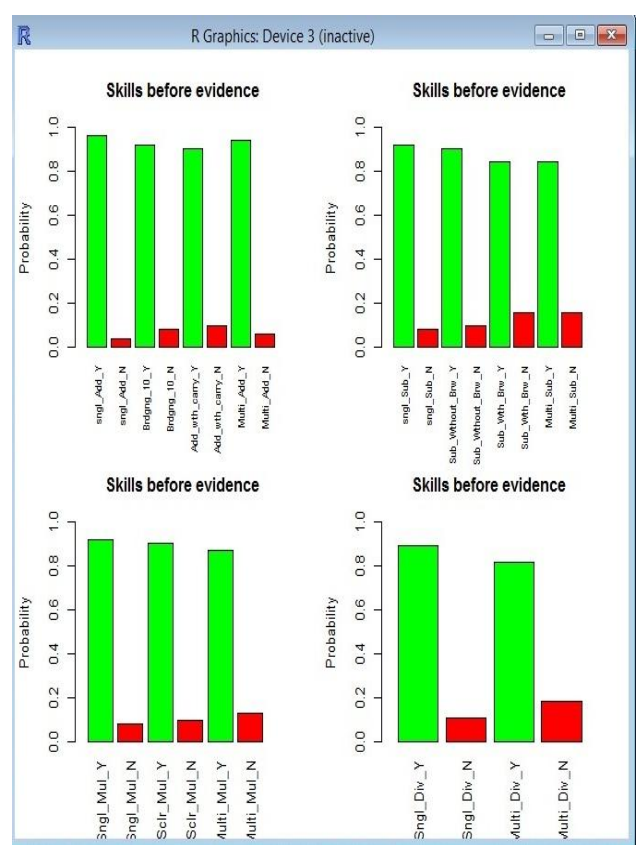

Figure 1: Individual skills before evidence

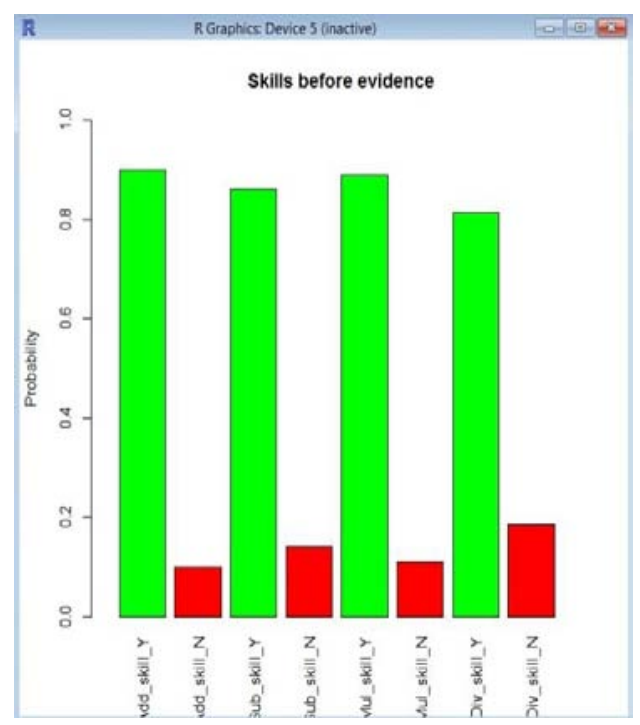

Figure 2: Overall skills before evidence

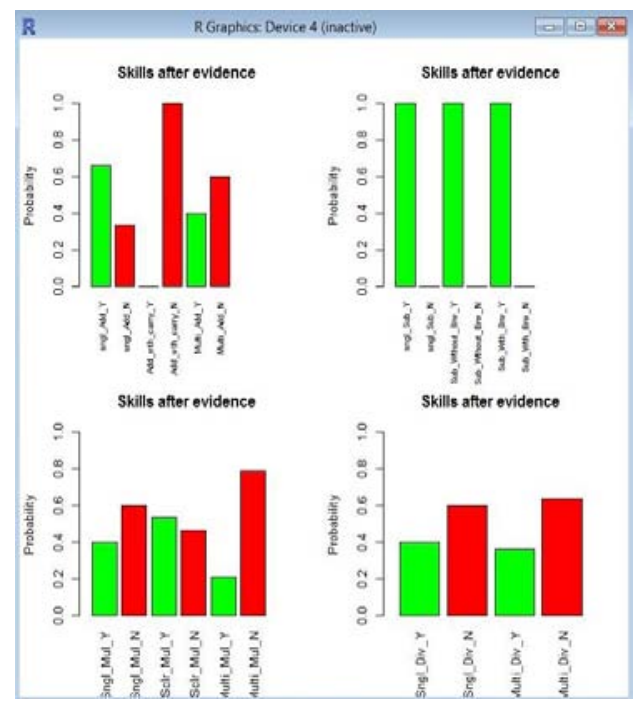

Figure 3: Individual skills after evidence

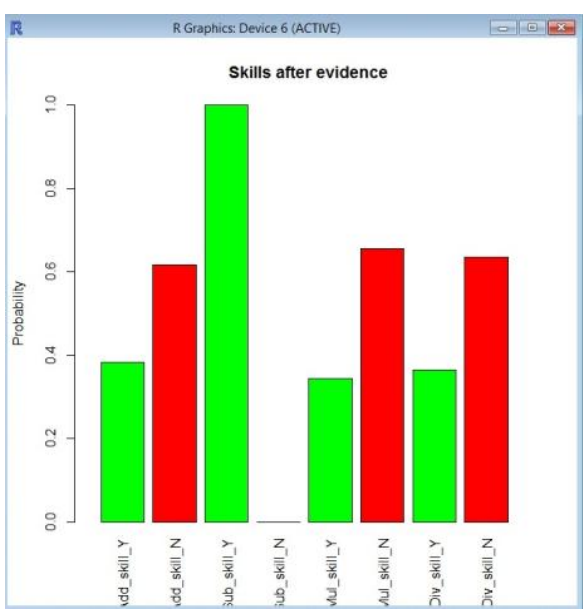

Figure 4: Overall skills after evidence

\section{Conclusion}

This study represents a model of intellectual process for improving arithmetic skills. The student model has the ability to be more refined by including additional available experimental data. The possibility to go back to easier skills importantly reduces an overall increase in learning rate. To conclude, our results show that modeling skills is beneficial and easy to use. In future we plan to increase the size of our model to capture more statistics. We would like to analyze the skills and their parameters in detail.

\section{References}

[1] Kaser, T., Baschera, G.M., Busetto, A.G., Klingler, S., Solenthaler, B., Buhmann, J.M., Gross, M.: Towards a Framework for Modelling Engagement Dynamics in Multiple Learning Domains. IJAIED (2012)

[2] Kaser, T., Baschera, G.M., Kohn, J., Kucian, K., Richtmann, V., Grond, U., Gross, M., von Aster, M.: Design and evaluation of the computer-based training program Calcularis for enhancing numerical cognition. Front. Psychol. (2013)

[3] Kaser, T., Busetto, A.G., Solenthaler, B., Baschera, G.M., Kohn, J., Kucian, K., von Aster, M., Gross, M.: Modelling and Optimizing Mathematics Learning in Children. IJAIED (2013)

[4] Kaser, T., Schwing, A.G., Hazan, T., Gross, M.: Computational Education using Latent Structured Prediction. To appear in Proc. AISTATS (2014)

[5] Ani Grubisic, Slavomir Stankov, Ivan Peraic, M: Ontology based approach to student model design. 